\title{
Analysis of permeate pressure build-up effects on separation performance of asymmetric hollow fiber membranes
}

\begin{abstract}
The study rectifies some perceptions about pressure build-up in hollow fiber membranes. It is a general intuition that operating at higher pressures permeates more gases, and sometimes the membrane module is tested or characterized at lower pressures to reduce gas consumption. It is also perceived that higher pressure build-up occurs at higher feed pressures, and membrane performance deteriorates at higher feed pressures. In this study, the apparent and intrinsic permeances of $\mathrm{H} 2$ and $\mathrm{N} 2$ in asymmetric cellulose acetate-based hollow fiber membranes were evaluated from gas permeation experiments and numerical analysis. It was shown that though the permeate pressure build-up increases as the feed pressure increases, the effect of the permeate pressure build-up on the membrane performance is actually reduced at higher feed pressures. Membrane performs close to its intrinsic separation properties if it is operated at high feed pressures, under which conditions the effect of pressure build-up on the membrane performance is minimized. The pressure build-up effect was further investigated by evaluating the percentage loss in the driving force due to permeate pressure build-up, and it was found that percentage loss in driving force is less at higher feed pressures than that at lower feed pressures.
\end{abstract}

Keyword: Membranes; Separations; Hollow fiber; Pressure build-up; Potted length; Permeance 